\title{
SUPERNOVA FRAGMENTS AND THE ORIGIN OF THE RAPID X-RAY VARIABILITY
}

\author{
R. CID FERNANDES \\ Institute of Astronomy, Cambridge, U.K. \\ R. TERLEVICH \\ Royal Greenwich Observatory, Cambridge, U.K. \\ G. TENORIO-TAGLE \\ Instituto de Astrofísica de Canarias, Tenerife, Spain \\ J. FRANCO \\ Instituto de Astronomia UNAM, México D. F., México \\ and \\ M. ROZYCZKA \\ Warsaw University Observatory, Warszaw, Poland
}

\begin{abstract}
The Starburst model for Radio Quiet Active Galactic Nuclei proved able to explain the origin of the broad line region, the variability characteristics of line and continuum in Seyfert galaxies, X-ray spectra, the luminosity function of QSOs and etc. But can we understand the rapid X-ray variability observed in several AGN with supernovae?
\end{abstract}

Key words: Active Galaxies, Starburst, Supernova Remnants, X-ray

In the Starburst model of AGN, strongly radiative compact supernova remnants (cSNRs) are the source of the broad emission lines, the high energy continuum and optical-UV variability. There is growing evidence that both the SN ejecta and its circumstellar medium are very clumpy and inhomogeneous. We have examined the role played by inhomogeneities in the SN ejecta on the evolution of otherwise normal cSNRs. The hydrodynamical models show the evolution of fragments into dense, fast moving "tortillas" (or "pancakes"), as they are processed by the reverse shock. The high densities and small cooling times result in isothermal shocks leading to large compression factors along the direction of motion. Dense fragments are not significantly decelerated by the reverse shock. They fly through the hot cavity between the reverse and outer shocks to ram against the thin shell at the outer edge of the remnant, producing a short, luminous burst of X-ray emission.

Our preliminary results indicate that a collection of pancake-shell collisions is capable of producing light curves and power spectra similar to those observed in AGN. Details of our computations will be presented elsewhere (Rozyczka et al. and Terlevich et al. 1993-submitted).

As a test of this model we encourage observers to look for short term X-ray variability in bona fine cSNRs. Also, high spatial resolution radio monitoring of the nuclei of nearby AGN should be able to detect individual supernovae as they go off in the nuclear cluster. This is a decisive observational test on the nature of the central engine.

T. J.-L. Courvoisier and A. Blecha: Multi-Wavelength Continuum Emission of AGN, 371.

(C) 1994 IAU. Printed in the Netherlands. 